\title{
The influence of tea catechins on immune function in the elderly: a single-arm, open-label clinical trial
}

\author{
Daisuke Furushima ${ }^{1}$, Ryo Iketani ${ }^{1}$, Takuma Nishimura ${ }^{1}$, Yui Hirama ${ }^{2}$, Shintaro Onishi ${ }^{2}$, \\ Takuya Mori ${ }^{2}$, Yuko Ohno ${ }^{3}$, Hiroshi Yamada ${ }^{1}$
}

\author{
${ }^{I}$ Department of Drug Evaluation \& Informatics, Graduate School of Pharmaceutical Sciences, University of Shizuoka, \\ Japan, ${ }^{2}$ Biological Science Laboratories, Kao Corporation, Japan, ${ }^{3}$ Department of Mathematical Health Science, \\ Course of Health Science, Graduate School of Medicine, Osaka University, Japan
}

\section{Background}

Immunosenescence is a biological phenomenon that occurs with aging. It is related to the occurrence of several diseases including infection, cancer, and dementia. Therefore, it is important to enhance or maintain immune function in the elderly to prevent such diseases. Previous in vivo studies reported that tea catechins likely enhance immune functions. We conducted a single-arm, open-label clinical trial using tea catechin beverages for two weeks to investigate the influence of tea catechins on immune function in the elderly.

\section{Methods}

We recruited 20 subjects aged 65 years or older without any disease that affects the immune function. They were prohibited from consuming teas and diets containing catechins from one week before the start of the intervention until the end of the trial. Participants consumed a beverage containing approximately $540 \mathrm{mg}$ of tea catechins in the morning once a day for two weeks. To evaluate the influence of tea catechins on immune function, natural killer (NK) cell activity (\%) was measured before and after intervention. The change in activity was expressed as the mean (95\% confidence interval), and statistical significance was determined using paired t-tests. The Pearson's correlation coefficient ( $\mathrm{r}$ ) was also calculated to investigate the correlation between NK cell activity before intervention and changes in NK cell activity. Results

Twenty subjects who fulfilled the eligibility criteria participated in and completed this study without any adverse events. All subjects consumed the beverage every day for two weeks. The corresponding change in NK cell activity was 17.1 (9.7 to 24.5) and this was statistically significant. Moreover, NK cell activity before intervention was significantly correlated with the ratio of posterior activity and prior activity $(\mathrm{r}=-0.63)$.

Conclusions

Although further studies using a parallel group randomized design are required to clarify the influence of tea catechins on immune function, our results suggest that the consumption of these compounds enhances or maintains NK cell activity in the elderly. The influence of catechins on NK activity was particularly pronounced in subjects with relatively low NK cell activity before intervention. Thus, daily catechin consumption might contribute to the enhancement of immune functions in the elderly. 